Pacific Journal of Mathematics

TORSION THEORIES AND RINGS OF QUOTIENTS OF 


\title{
TORSION THEORIES AND RINGS OF QUOTIENTS OF MORITA EQUIVALENT RINGS
}

\section{DARRELL R. TURNIDGE}

\begin{abstract}
A ring of left quotients $Q_{\mathscr{T}}$ of a ring $R$ can be constructed relative to any hereditary torsion class $\mathscr{T}$ of left $R$-modules. For Morita equivalent rings $R$ and $S$ we construct a one-toone correspondence between the hereditary torsion classes (strongly complete Serre classes) of ${ }_{R} \mathfrak{M}$ and ${ }_{s} \mathfrak{M}$ and describe the resulting correspondence between the strongly complete filters of left ideals of $R$ and $S$. We show that the proper rings of left quotients of $R$ and $S$ relative to corresponding hereditary torsion classes are Morita equivalent. Applications are made to the maximal and the classical rings of left quotients and the corresponding torsion theories.
\end{abstract}

A torsion theory for the category ${ }_{R} \mathfrak{M}$ of unitary left modules over an associative ring $R$ with identity has been defined by Dickson [3] to be a pair $(\mathscr{T}, \mathscr{F})$ of classes of left $R$-modules such that

(a) $\mathscr{T} \cap \mathscr{F}=\{0\}$

(b) $\mathscr{T}$ is closed under homomorphic images

(c) $\mathscr{F}$ is closed under submodules

(d) for every left $R$-module $M$ there exists a submodule $T(M)$ of $M$ with $T(M) \in T$ and $\mathrm{M} / T(M) \in \mathscr{F}$.

A class $\mathscr{T}(\mathscr{F})$ of left modules is called a torsion (torsion-free) class if there is a (necessarily unique) class $\mathscr{F}(\mathscr{T})$ such that $(\mathscr{T}, \mathscr{F})$ is a torsion theory. A torsion class closed under submodules is said to be hereditary. By [3, Theorem 2.3] a class $\mathscr{T}$ is a hereditary torsion class if and only if it is closed under submodules, homomorphic images, extensions, and arbitrary direct sums. Walker and Walker [13] call such a class a strongly complete Serre class. Gabriel [4] has shown that for a ring $R$ there is a one-to-one correspondence between the strongly complete Serre classes of ${ }_{R} \mathfrak{M}$ and the strongly complete filters $F$ of left ideals of $R$ given by the mapping

$$
\mathscr{T} \longrightarrow F(\mathscr{T})=\{I \leqq R \mid R / I \in \mathscr{T}\}
$$

where $I \leqq R$ denotes that $I$ is a left ideal of $R$. The inverse correspondence is given by

$$
F \longrightarrow \mathscr{T}(F)=\left\{M \in_{R} \mathfrak{M} \mid(0: m) \in F \text { for all } m \in M\right\}
$$

where $(0: m)=\{r \in R \mid r m=0\}$. We say a strongly complete filter $F$ of left ideals of $R$ is faithful if $(0: r) \in F$ implies $r=0$ for each $r \in$ $R$. A strongly complete Serre class $\mathscr{T}$ is called a faithful Serre 
class if $F(\mathscr{T})$ is faithful. Viewing $\mathscr{T}$ as a hereditary torsion class this is equivalent to the requirement that ${ }_{R} R$ is torsion-free.

1. Rings of quotients. Throughout this section $\mathscr{T}$ will denote a faithful Serre class of ${ }_{R} \mathfrak{M}$ with associated filter $F$. Then $(\mathscr{T}, \mathscr{F})$ is a torsion theory for ${ }_{R} \mathfrak{M}$ and ${ }_{R} R \in \mathscr{F}$ where

$$
\mathscr{F}=\left\{M \in_{R} \mathfrak{M} \mid \operatorname{Hom}_{R}(T, M)=0 \text { for all } T \in \mathscr{T}\right\} \text {. }
$$

Let $\mathscr{A}$ denote the quotient category of ${ }_{R} \mathfrak{M}$ relative to $\mathscr{T}$ as defined in [4] and let

$$
R_{-}=\operatorname{Hom}_{\varkappa}(R, R)=\underset{I \in F}{\stackrel{\lim }{\longrightarrow}} \operatorname{Hom}_{R}(I, R)
$$

the endomorphism ring of $R$ as an object of $\mathscr{A}$. The opposite ring of $R$, is denoted by $Q$, and is called the ring of left quotients of $R$ relative to $\mathscr{T}$. The natural ring anti-isomorphism of $R$ and $\operatorname{Hom}_{R}(R, R)$ induces a one-to-one ring homomorphism $\varphi: R \rightarrow Q_{\sigma}$. We usually identify $R$ as a unital subring of $Q_{5}$. More generally, for each left $R$-module $M$ let

$$
M_{\nearrow}=\operatorname{Hom}_{\searrow}(R, M)=\underset{R / I, M M^{\prime} \in \mathscr{S}}{\lim } \operatorname{Hom}_{R}\left(I, M / M^{\prime}\right) .
$$

Using the composition of morphisms in $\mathscr{A}$ each $M_{\varnothing}$ is a right $R_{8}$ module and thus a left $Q$-module. The ring homomorphism $\varphi$ induces a left $R$-module structure on $M_{\mathscr{T}}$ and there is a natural left $R$-homomorphism $\varphi_{M}: M \rightarrow M_{\sigma}$ given by $\varphi_{M}(m)=\left[\rho_{m}\right]$, the equivalence class of $\rho_{m}$ in $M_{\sigma}$, where for each $m \in M, \rho_{m}: R \rightarrow M$ by $\rho_{m}(r)=r m$. As shown in [13] for each left $R$-module $M$, $\operatorname{ker} \varphi_{M}=T(M)=\{m \in M \mid(0: m) \in F\}$.

A left $R$-module $M$ is said to be $\mathscr{T}$-injestive if for every exact sequence

$$
0 \longrightarrow K \longrightarrow L \longrightarrow T \longrightarrow 0
$$

of left $R$-modules with $T \in \mathscr{T}$, the associated sequence

$$
0 \longrightarrow \operatorname{Hom}_{R}(T, M) \longrightarrow \operatorname{Hom}_{R}(L, M) \longrightarrow \operatorname{Hom}_{R}(K, M) \longrightarrow 0
$$

is exact. By [13, Proposition 4.2] for each left $R$-module $M$

$$
E_{\mathscr{J}}(M)=\{x \in E(M) \mid(M: x) \in F\}
$$

is $\mathscr{T}$-injective and is (up to isomorphism) the unique minimal $\mathscr{T}$-injective module containing $M$ where $E(M)$ is an injective envelope of $M$. We call $E_{-}(M)$ a $\mathscr{T}$-injestive envelope of $M$. The following lemmas are consequences of [4, Proposition 4, page 413] but the proof is included for the sake of completeness. 
Lemma 1.1. For each $M \in \mathscr{F}, E_{\mathscr{J}}(M) \cong M_{\Im}$ as left $R$-modules.

Proof. For each $x \in E_{\mathscr{S}}(M),(M: x) \in F$. Define $\lambda: E_{\mathscr{S}}(M) \rightarrow M_{\mathscr{S}}$ by $\lambda(x)=\left[\rho_{x}\right]$ for each $x \in E_{\sigma}(M)$ where $\rho_{x}(r)=r x$ for each $r \in(M: x)$. It is easily checked that $\lambda$ is additive.

By [3, Theorem 2.9] $\mathscr{F}$ is closed under injective envelopes. Thus $E(M)$ and hence $E_{\mathscr{J}}(M) \in \mathscr{F}$. If $x \in E_{\mathscr{J}}(M)$ and $\lambda(x)=0$, then $I x=$ 0 for some $\mathrm{I} \in F$. Since $E_{\mathcal{J}}(M) \in \mathscr{F}$ this implies $x=0$. Thus $\lambda$ is one-to-one.

Let $[f] \in M_{\mathscr{S}}$ be represented by $f: I \rightarrow M$ with $I \in F$. Since $E_{\mathscr{S}}(M)$ is $\mathscr{T}$-injective and contains $M, f$ extends to an $R$-homomorphism $\bar{f}: R$ $\rightarrow E_{\mathscr{J}}(M)$. Let $x=\bar{f}(1) \in E_{\mathscr{J}}(M)$. Then $\lambda(x)=[f]$ so $\lambda$ is onto.

Finally, for $x \in E_{\mathscr{J}}(M)$ and $r \in R$ one checks that $\lambda(r x)=r \lambda(x)$.

In the special case that $M={ }_{R} R$ we have the following.

Lemma 1.2. As left $R$-modules, $Q_{\nearrow} \cong E_{\mathscr{J}}(R)$.

From this we get the following proposition which will be used later in studying Morita equivalence of quotient rings.

Proposition 1.3. If $\mathscr{T}$ is any faithful Serre class of ${ }_{R} \mathfrak{M}$, then $Q, \cong \operatorname{End}_{R}\left(E_{-}(R)\right)^{0}$ as rings.

Proof. Let $f \in \operatorname{End}_{R}\left(Q_{-}\right)$and let $q, x \in Q_{\sigma}$. Then for each $r \in(R: q)$, $r(q f(x)-f(q x))=0$. But $\left(R: q ; \in F\right.$ and $Q_{,} \in \mathscr{F}$. Thus $q f(x)=f(q x)$. It follows that $\operatorname{End}_{R}\left(Q_{,}\right)=\operatorname{End}_{Q_{\sigma}}\left(Q_{\digamma}\right)$. Using the natural ring antiisomorphism and (1.2) we have

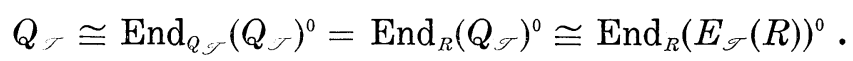

We now investigate more closely the relationship between the ring of left quotients $Q_{J}$ and the torsion theory $(\mathscr{T}, \mathscr{F})$. As previously noted $\operatorname{ker} \varphi_{M}=T(M)$ for each left $R$-module $M$ where $\varphi_{M}$ is the natural $R$-homomorphism from $M$ to $M$. For each left $R$-module $M, \varphi_{M}=\theta_{M} \eta_{M}$ where

$$
\eta_{M}: M \longrightarrow Q_{\mathcal{S}} \otimes_{R} M \quad \text { by } \quad \eta_{M}(m)=1 \otimes m
$$

and

$$
\theta_{M}: Q_{\mathscr{S}} \otimes_{R} M \longrightarrow M_{\mathscr{S}} \text { by } \theta_{M}(x \otimes m)=x \varphi_{M}(m)
$$

for each $m \in M$ and each $x \in Q_{\sigma}$. Thus in general we have ker $\eta_{M} \subseteq$ $T(M)$.

THEOREM 1.4. Let $\mathscr{T}$ be a strongly complete Serre class of ${ }_{R} \mathfrak{M}$. 
Then $T(M)=\operatorname{ker} \eta_{M}$ for every left $R$-module $M$ if and only if $Q_{\sigma} \varphi(I)=$ $Q_{\sigma}$ for all $I \in F=F(\mathscr{T})$. Moreover $Q_{\sigma}$ is flat as a right $R$-module whenever $T(M)=\operatorname{ker} \eta_{M}$ for all $M$.

Proof. If $Q, \varphi(I)=Q_{\triangle}$ for all $I \in F$, then $\theta_{M}$ is an isomorphism for each left $R$-module $M$ by [13, Theorem 3.2]. Hence $\operatorname{ker} \varphi_{M}=\operatorname{ker}$ $\eta_{M}=T(M)$ for every $M$.

Conversely if ker $\eta_{M}=T(M)$ for every left $R$-module $M$, then $R / I=$ ker $\eta_{R / I}$ for each $I \in F$. Thus $Q . \bigotimes_{R} R / I=0$ for every $I \in F$. Hence for each $I \in F$ the mapping $Q_{\circlearrowleft} \bigotimes_{R} I \longrightarrow Q_{\odot} \bigotimes_{R} R$ is an isomorphism. Thus $Q_{\sigma}=Q_{\odot} \varphi(I)$ for each $I \in F$. The last remark follows. by [13, Corollary 3.3].

We conclude this section indicating two important special cases of this result.

A left ideal $I$ of $R$ is said to be dense if $(I: a) b \neq 0$ for all $a, b$ in $R$ with $b \neq 0$. The strongly complete faithful filter $D$ of dense left ideals of $R$ is maximal among all the strongly complete faithful filters of left ideals of $R$. The corresponding faithful Serre class

$$
\mathscr{T}^{\prime}=\left\{M \in_{R} \mathfrak{M} \mid(0: m) \in D \text { for all } m \in M\right\}
$$

is thus maximal among all the faithful Serre classes of ${ }_{R} \mathfrak{M}$ and coincides with the $E(R)$-torsion class considered by Jans [6]. The ring of left quotients of $R$ relative to $\mathscr{T}^{\prime}$ is called the maximal ring of left quotients of $R$ and is denoted by $Q\left({ }_{R} R\right)$.

For each left $R$-module ${ }_{R} M$ we let $Z\left({ }_{R} M\right)$ denote the set of all elements of ${ }_{R} M$ whose annihilator is an essential left ideal of $R$. Then $Z\left({ }_{R} M\right)$ is a submodule of ${ }_{R} M$ called the singular submodule of ${ }_{R} M$. For a ring $R$ with $Z\left({ }_{R} R\right)=0$, a left ideal is dense if and only if it is essential. For such rings $Q\left({ }_{R} R\right)$ is von Neumann regular. (See [7]) Moreover for a ring $R$ with $Z\left({ }_{R} R\right)=0, Q\left({ }_{R} R\right)$ is semisimple (with minimum condition) if and only if $Q\left({ }_{R} R\right) I=Q\left({ }_{R} R\right)$ for all essential left ideals of $R$ by [11, Theorem 1.6] or [13, Theorem 4.19]. Combining these facts with (1.4) we get the following results of Sandomierski [11].

Proposition 1.5. Let $R$ be a ring with $Z\left({ }_{R} R\right)=0$. Then $Z(M)=$ ker $\eta_{M}$ where $\eta_{M}: M \longrightarrow Q\left({ }_{R} R\right) \otimes_{R} M$ via $\eta_{M}(m)=1 \otimes m$ for every left $R$-module $M$ if and only if $Q\left(_{R} R\right)$ is semisimple. Moreover, if $Q\left(_{R} R\right)$ is semisimple it is flat as a right $R$-module.

Let $U$ denote the set of two-sided nonzero divisors of $R$, let $F_{C}=$ $\{I \leqq R \mid I \cap U \neq \varnothing\}$ and let

$$
\mathscr{T}_{C}=\left\{M \in_{R} \mathfrak{M} \mid(0: m) \in F_{C} \text { for all } m \in M\right\} \text {. }
$$

A ring $R$ is said to be left Ore if for all $a \in R$ and $d \in U$ there exist. 
$a^{\prime} \in R$ and $d^{\prime} \in U$ such that $d^{\prime} a=a^{\prime} d$. One checks that $F_{C}$ is a strongly complete faithful filter of left ideals of $R$ and $\mathscr{T}_{C}$ is a faithful Serre class of ${ }_{R} \mathfrak{M}$ if and only if $R$ is left Ore. For any left Ore ring $R$, the ring of left quotients of $R$ relative to $\mathscr{T}_{C}$ is denoted by $Q_{C}(R)$ and is called the classical ring of left quotients of $R$. For a left Ore ring $R, Q_{C}(R)$ has the following properties:

(a) $d \in U$ implies $d^{-1}$ exists in $Q_{C}(R)$

(b) for each $q \in Q_{C}(R)$, there exists $a \in R$ and $d \in U$ with $q=d^{-1} a$. For a left Ore ring $R$, every $I \in F_{C}$ contains an invertible element of $Q_{C}(R)$. Hence $Q_{C}(R) I=Q_{C}(R)$ for every $I \in F_{C}$. Applying (1.4) we have the following results of Levy [8].

Proposition 1.6. Let $R$ be a left Ore ring. Then for each left $R$-module $M$, the kernel of the mapping $\eta_{M}: M \longrightarrow Q_{C}(R) \bigotimes_{R} M$ defined by $\eta_{M}(m)=1 \otimes m$ is $T_{C}(M)=\left\{m \in M \mid(0: m) \in F_{C}\right\}$. Moreover $Q_{C}(R)$ is flat as a right $R$-module.

2. Morita equivalence of quotient rings. Morita has shown that two rings $R$ and $S$ have equivalent categories of unitary left modules if and only if $S \cong \operatorname{End}_{R}\left(P_{R}\right)$ for some right $R$-progenerator $P_{R}$ where a right $R$-module $P_{R}$ is called a progenerator if it is finitely generated projective and if the right regular module $R_{R}$ is isomorphic to a direct summand of a direct sum of copies of $P_{R}$. (See [1] or [10]) Two such rings are said to be Morita equivalent. Throughout this paper we assume $S=\operatorname{End}_{R}\left(P_{R}\right)$ with $P_{R}$ a progenerator. Then the functors

$$
G=P \bigotimes_{R}(\quad):{ }_{R} \mathfrak{M} \longrightarrow{ }_{S} \mathfrak{M}
$$

and

$$
H=P^{*} \bigotimes_{S}(\quad):{ }_{S} \mathfrak{M} \longrightarrow{ }_{R} \mathfrak{M}
$$

are inverse category equivalences where $P^{*}=\operatorname{Hom}_{R}(P, R)$ is a left $R$ progenerator.

If $\mathscr{T}(R)$ is any strongly complete Serre class of ${ }_{R} \mathfrak{M}$, then

$$
\mathscr{T}(S)=\{M \in \mathfrak{M} \mid H(M) \in \mathscr{T}(R)\}
$$

is a strongly complete Serre class of ${ }_{s} \mathfrak{M}$ since $H$ preserves exactness and direct sums. The mapping pairing each $\mathscr{T}(R)$ with $\mathscr{T}(S)$ as defined above gives a one-to-one correspondence between the strongly complete Serre classes of ${ }_{R} \mathfrak{M}$ and ${ }_{S} \mathfrak{M}$. Henceforth $\mathscr{T}(R)$ and $\mathscr{T}(S)$ will denote corresponding strongly complete Serre classes of ${ }_{R}^{\mathfrak{M}}$ and $s_{\mathfrak{M}}$ respectively. By our introductory remarks there are (unique) classes $\mathscr{F}(R)$ and $\mathscr{F}(S)$ such that $(\mathscr{T}(R), \mathscr{F}(R))$ and $(\mathscr{T}(S), \mathscr{F}(S))$ are hereditary torsion theories for ${ }_{R} \mathfrak{M}$ and ${ }_{S} \mathfrak{M}$ respectively. Moreover, 


$$
\mathscr{F}(S)=\left\{M \in{ }_{S} \mathfrak{M} \mid H(M) \in \mathscr{F}(R)\right\}
$$

Proposition 2.1. $\mathscr{T}(R)$ is faithful if and only if $\mathscr{T}(S)$ is faithful.

Proof. If $\mathscr{T}(R)$ is faithful, then ${ }_{R} R \in \mathscr{F}(R)$. Hence by [3, Theorem 2.3] every finitely generated projective left $R$-module is in $\mathscr{F}(R)$. But $H\left({ }_{S} S\right) \cong{ }_{R} P^{*}$ is a finitely generated projective left $R$ module, so $H\left({ }_{S} S\right) \in \mathscr{F}(R)$. Thus ${ }_{S} S \in \mathscr{F}(S)$, so $\mathscr{T}(S)$ is faithful. The converse follows by a dual argument.

Throughout the remainder of this paper unless otherwise noted we restrict our attention to the case where $\mathscr{T}(R)$ and $\mathscr{T}(S)$ and faithful.

We let $Q_{\mathscr{T}(R)}$ and $Q_{\mathscr{T}(S)}$ denote the rings of left quotients of $R$ and $S$ relative to $\mathscr{T}(R)$ and $\mathscr{T}(S)$ respectively as defined in $\S 1$. Before examining the Morita equivalence of $Q_{S(R)}$ and $Q_{\mathscr{S}(S)}$ we need a few observations on $\mathscr{T}$-injectivity. Using routine arguments with the category equivalences $G$ and $H$ one gets the following.

Lemma 2.2. Let $M$ be a left $R$-module. Then $M$ is $\mathscr{T}(R)-$ injective if and only if $G(M)$ is $\mathscr{T}(S)$-injective.

Proposition 2.3. Let $M$ be a left $R$-module with $\mathscr{T}(R)$-injective envelope $E_{\mathscr{T}(R)}(M)$. Then $G\left(E_{\mathscr{T}_{(R)}}(M)\right.$ is a $\mathscr{T}(S)$-injective envelope of $G(M)$.

Proof. By the lemma, $G\left(E_{\mathscr{T}(R)}(M)\right)$ is a $\mathscr{T}(S)$-injective extension of $G(M)$. Using the fact that $G$ induces an isomorphism between the lattices of submodules of $E_{T_{(R)}}(M)$ and $G\left(E_{\sigma(R)}(M)\right)$ one checks that $G\left(E_{\mathscr{T}(R)}(M)\right)$ is a minimal $\mathscr{T}(S)$-injective extension of $G(M)$.

Two left $R$-modules $M$ and $N$ are said to be similar if each is isomorphic to a direct summand of a finite direct sum of copies of the other. Observing that finite direct sums of $\mathscr{T}(R)$-injective modules are $\mathscr{T}(R)$-injective one checks that similar left $R$-modules have similar $\mathscr{T}(R)$-injective envelopes. Since the left $R$-module ${ }_{R} P^{*}$ is a progenerator and is thus similar to ${ }_{R} R$ we have $E_{\sigma(R)}\left({ }_{R} P^{*}\right)$ is similar to $E_{\mathcal{J}(R)}\left({ }_{R} R\right)$.

To simplify our notation we let $E_{\mathscr{T}}(R)=E_{\mathscr{T}(R)}\left({ }_{R} R\right), E_{\sigma}\left(P^{*}\right)=$ $E_{\mathscr{T}(R)}\left({ }_{R} P^{*}\right)$ and $E_{\mathscr{T}}(S)=E_{\mathscr{T}(S)}\left({ }_{S} S\right)$. Then using (2.3) and the fact that $G\left(P^{*}\right) \cong{ }_{S} S$, we have

$$
\begin{aligned}
\operatorname{End}_{R}\left(E_{\sigma}\left(P^{*}\right)\right) & \cong \operatorname{End}_{S}\left(G\left(E_{\mathscr{\sigma}}\left(P^{*}\right)\right)\right) \\
& \cong \operatorname{End}_{S}\left(E_{\mathscr{\sigma}}\left(G\left(P^{*}\right)\right)\right) \\
& \cong \operatorname{End}_{S}\left(E_{\mathscr{J}}(S)\right) .
\end{aligned}
$$


Thus by (1.3)

$$
Q_{\mathscr{\sigma}(R)} \cong \operatorname{End}_{R}\left(E_{\mathscr{T}}(R)\right)^{0}
$$

and

$$
Q_{\mathscr{\sigma}(S)} \cong \operatorname{End}_{S}\left(E_{\mathscr{J}}(S)\right)^{0} \cong \operatorname{End}_{R}\left(E_{\mathscr{J}}\left(P^{*}\right)\right)^{0} .
$$

Hirata [5, Theorem 1.5] has shown that for similar left $R$-modules $M$ and $N$, the rings $E=\operatorname{End}_{R}(M)^{0}$ and $E^{\prime}=\operatorname{End}_{R}(N)^{0}$ are Morita equivalent. (The opposite rings arise from our convention of regarding mappings as operating on the left.) Moreover $\operatorname{Hom}_{R}(M, N)$ is a progenerator both as a left $E$-module and as a right $E^{\prime}$-module. Similarly $\operatorname{Hom}_{R}(N, M)$ is a progenerator both as a left $E^{\prime}$-module and as a right $E$-module.

Letting $M=E_{\mathscr{T}}(R)$ and $N=E_{\mathscr{T}}\left(P^{*}\right)$ we conclude that the rings $Q_{\sigma(R)}$ and $Q_{\sigma(S)}$ are Morita equivalent and that $\operatorname{Hom}_{R}\left(E_{\mathscr{T}}\left(P^{*}\right), E_{\mathscr{J}}(R)\right)$ is a progenerator both as a left $Q_{\mathscr{T}(S)}$-module and as a right $Q_{\mathscr{S}(R)^{-}}$ module.

Since $P \bigotimes_{R} E_{\mathscr{J}}(R)$ is $\mathscr{T}(S)$-injective and

$$
0 \longrightarrow S \longrightarrow E_{\mathscr{J}}(S) \longrightarrow E_{\mathscr{J}}(S) / S \longrightarrow 0
$$

is an exact sequence of left $S$-modules with $E_{\mathscr{J}}(S) / S \in \mathscr{T}(S)$,

$$
\begin{gathered}
0 \longrightarrow \operatorname{Hom}_{S}\left(E_{\Im}(S) / S, P \bigotimes_{R} E_{\digamma}(R)\right) \longrightarrow \operatorname{Hom}_{S}\left(E_{\mathscr{J}}(S), P \bigotimes_{R} E_{\mathscr{J}}(R)\right) \\
\longrightarrow \operatorname{Hom}_{S}\left(S, P \bigotimes_{R} E_{\mathscr{J}}(R)\right) \longrightarrow 0
\end{gathered}
$$

is an exact sequence of right $Q_{\mathscr{T}(R)}$-modules. But $\mathrm{Hom}_{S}\left(E_{\mathscr{\sigma}}(S) / S\right.$, $\left.P \bigotimes_{R} E_{\mathscr{T}}(R)\right)=0$ since $E_{\mathscr{T}}(S) / S \in \mathscr{T}(S)$ and $P \bigotimes_{R} E_{\mathscr{T}}(R) \in \mathscr{F}(S)$. Hence as a right $Q_{\mathscr{T}(R)}$-module

$$
\begin{aligned}
& \operatorname{Hom}_{R}\left(E_{\mathscr{J}}\left(P^{*}\right), E_{\mathscr{J}}(R)\right) \cong \operatorname{Hom}_{S}\left(E_{\mathscr{J}}(S), P \otimes_{R} E_{\mathscr{J}}(R)\right) \\
& \cong \operatorname{Hom}_{S}\left(S, P \otimes_{R} E_{\mathscr{T}}(R)\right) \\
& \cong P \bigotimes_{R} E_{\mathscr{T}}(R) \cong P \bigotimes_{R} Q_{\mathscr{T}(R)} \text {. }
\end{aligned}
$$

Summarizing, we have the following theorem.

THeOREm 2.4. Let $\mathscr{T}(R)$ be a faithful Serre class of ${ }_{R} \mathfrak{M}$ and let $\mathscr{T}(S)$ be the corresponding faithful Serre class of $\mathrm{M}$. Then the rings of left quotients $Q_{\sigma_{(R)}}$ and $Q_{S_{(S)}}$ are Morita equivalent. Moreover $P \otimes_{R} Q_{\sigma(R)}$ is a right $Q_{\widetilde{\sigma}(R)}$-progenerator with

$$
Q_{\Im(S)} \cong \operatorname{End}_{Q_{\mathscr{S}(R)}}\left(P \bigotimes_{R} Q_{\mathscr{T}(R)}\right) \text {. }
$$

Let $F_{R}$ be a free right $R$-module of rank $n$. Then $\operatorname{End}_{R}\left(F_{R}\right) \cong R_{n}$ and $\operatorname{End}_{Q \mathscr{T}(R)}\left(F \bigotimes_{R} Q_{\mathscr{S}(R)}\right) \cong\left(Q_{\mathscr{T}(R)}\right)_{n}$.

Corollary 2.5. Let $\mathscr{T}(R)$ be a faithful Serre class of ${ }_{R} \mathfrak{M}$ and 
let $\mathscr{T}\left(R_{n}\right)$ be the corresponding faithfu? Serre class of ${ }_{R_{n}} \mathfrak{M}$. Then $Q_{\sigma\left(R_{n}\right)} \cong\left(Q_{\mathscr{T}(R)}\right)_{n}$.

Previously in this section we described a one-to-one correspondence between the strongly complete Serre classes of ${ }_{R} \mathfrak{M}$ and ${ }_{s} \mathfrak{M}$. We conclude this section by describing the resulting correspondence between the strongly complete filters of left ideals of $R$ and $S$.

By hypothesis $S=\operatorname{End}_{R}\left(P_{R}\right)$ with $P_{R}$ a progenerator. Since $P_{R}$ is finitely generated and projective, by the Dual Basis Lemma [2, Proposition VII, 3.1] there exist $x_{1}, \cdots, x_{n} \in P$ and $f_{1}, \cdots, f_{n} \in P^{*}$ such that

$$
x=\sum x_{i} f_{i}(x) \text { and } f=\sum f\left(x_{i}\right) f_{i}
$$

for all $x \in P$ and all $f \in P^{*}$.

For each left ideal $I$ of $R$, let

$$
\bar{I}=\left\{s \in S \mid s\left(x_{i}\right) \in P I \text { for all } i=1, \cdots, n\right\}=\cap\left(0:{ }_{s} \bar{x}_{i}\right)
$$

where $\bar{x}_{i}$ is the canonical image in $P / P I$ of $x_{i}$. Similarly, for each left ideal $J$ of $S$, let

$$
\bar{J}=\left\{r \in R \mid \varkappa f_{i} \in P^{*} J \text { for all } i=1, \cdots, n\right\}=\cap\left(0:_{R} \bar{f}_{i}\right)
$$

where $\bar{f}_{i}$ is the canonical image in $P^{*} / P^{*} J$ of $f_{i}$.

If $I \in F(R)$, the strongly complete filter of left ideals corresponding to $\mathscr{T}(R)$, then $G(R / I)=P \bigotimes_{R} R / I \cong P / P I \in \mathscr{T}(S)$. Thus $\left(0:_{s} \bar{x}_{i}\right) \in$ $F(S)$, the strongly complete filter of left ideals corresponding to $\mathscr{T}(S)$, for all $i=1, \cdots, n$. It follows that $\bar{I} \in F(S)$.

Similarly, if $J \in F(S)$, then $H(S / J)=P^{*} \bigotimes_{S} S / J \cong P^{*} / P^{*} J \in \mathscr{T}(R)$. Thus $\left(0:{ }_{R} \bar{f}_{i}\right) \in F(R)$ for all $i=1, \cdots, n$. Thus $\bar{J} \in F(R)$.

Finally, if $J \in F(S)$ and $I=\bar{J}$ one checks that $\bar{I} \leqq J$. Thus we have shown the following.

Proposition 2.6. Let $\mathscr{T}(R)$ and $\mathscr{T}(S)$ be corresponding strongly complete Serre classes of ${ }_{R} \mathfrak{M}$ and ${ }_{s} \mathfrak{M}$ with associated filters of left ideals $F(R)$ and $F(S)$ and let $J$ be a left idea! of $S$. Then $J \in F(S)$ if and only if there exists an $I \in F(R)$ with $\bar{I} \leqq J$.

3. Applications. In this section the results of the preceding section and applied to the maximal and the classical rings of left quotients.

Let $\mathscr{T}^{\prime}(R)$ and $\mathscr{T}^{\prime}(S)$ denote the maximal faithful Serre classes of $R_{R} \mathfrak{M}$ and $S_{S}$. By virtue of their maximality $\mathscr{T}^{\prime}(R)$ and $\mathscr{T}^{\prime}(S)$ correspond as in $\S 2$. Hence as a special case of (2.4) we have the following.

THEOREM 3.1. The maximal rings of left quotients of Morita 
equivalent rings are Morita equivalent.

CoRollary 3.2. Let $R$ and $S$ be Morita equivalent rings. Then $Q\left({ }_{R} R\right)$ is von Neumann regular if and only if $Q\left({ }_{S} S\right)$ is von Neumann regular. Consequently, $Z\left(_{R} R\right)=0$ if and only if $Z\left({ }_{S} S\right)=0$.

In the following let $R$ be a left Ore ring and let $\mathscr{T}_{C}(R)$ and $F_{C}(R)$ be as defined in $\S 1$. As usual let $S=\operatorname{End}_{R}\left(P_{R}\right)$ with $P_{R}$ a right $R$-progenerator. It is unknown whether $S$ is necessarily left Ore. Indeed, we do not know whether the ring of $n \times n$ matrices over a left Ore ring is left Ore for $n>1$ unless additional requirements are placed on $Q_{C}(R)$. (See Small [12, Theorem 2.28]) As a partial result we shall show that $S$ is left Ore if $R$ is commutative.

As indicated in $\S 2$,

$$
\mathscr{T}(S)=\left\{M \in{ }_{S} \mathfrak{M} \mid H(M) \in \mathscr{T}_{C}(R)\right\}
$$

is a faithful Serre class of $s^{\mathfrak{M}}$ with associated filter $F(S)$ given by

$$
F(S)=\left\{J \leqq S \mid \bar{I} \leqq J \text { for some } I \in F_{C}(R)\right\} .
$$

Let

$$
F_{C}(S)=\{J \leqq S \mid J \cap U(S) \neq \varnothing\}
$$

where $U(S)$ denotes the set of nonzero divisors of $S$ and let

$$
\mathscr{T}_{C}(S)=\left\{M \in \mathfrak{M} \mid(0: m) \in F_{C}(S) \text { for all } m \in M\right\} \text {. }
$$

If $\mathscr{T}_{c}(S)=\mathscr{T}(S)$ or equivalently if $F_{c}(S)=F(S)$, then $S$ is left Ore and $Q_{C}(R)$ and $Q_{C}(S)$ are Morita equivalent.

THEOREM 3.3. If $R$ is commutative, then $S$ is left Ore and $Q_{C}(R)$ and $Q_{C}(S)$ are Morita equivalent.

Proof. We show $F_{C}(S)=F(S)$. Let $J \in F(S)$. Then there exists $I \in F_{C}(R)$ with $\bar{I} \leqq J$. Let $d \in I \cap U(R)$ and define $\rho_{d} \in S$ by $\rho_{d}(x)=x d$ for each $x \in P$. Then $\rho_{d} \in \bar{I}$ since $\rho_{d}(x) \in P I$ for all $x \in P$. For all $s \in S$ and all $x \in P, \rho_{d} s(x)=s \rho_{d} s(x)=s(x) d$. If $\rho_{d} s=0$ then $f_{i}(s(x)) d=0$ for $i=1, \cdots, n$. Since $d \in U(R)$ and $f_{i}(s(x)) \in R$ this implies that $f_{i}(s(x))=0$ for $i=1, \cdots, n$. Therefore $s(x)=\sum x_{i} f_{i}(s(x))=0$ for all $x \in P$. Hence $s=0$ so $\rho_{d} \in U(S)$. Thus $\rho_{d} \in J \cap U(S)$ so $J \in F_{C}(S)$. Therefore $F(S) \subseteq F_{c}(S)$.

Conversely, let $J \in F_{C}(S)$ and let $s \in J \cap(S)$. Let $F_{R}$ be a free right $R$-module of rank $n$ with $F_{R}=P_{R} \oplus P_{R}{ }^{\prime}$ for some $P_{R}{ }^{\prime}$ and let $\Lambda: \operatorname{End}_{R}\left(F_{R}\right) \rightarrow R_{n}$ be a unital ring isomorphism. Using the fact that $P_{R}$ is a progenerator one checks that $\bar{s} \in \operatorname{End}_{R}\left(F_{R}\right)$ defined by $\bar{s}\left(p, p^{\prime}\right)=$ $\left(s(p), p^{\prime}\right)$ is a nonzero divisor of $\operatorname{End}_{R}\left(F_{R}\right)$. Since $\Lambda(\bar{s})$ is a nonzero 
divisor of $R_{n}$ and $R$ is commutative, $\operatorname{det} \Lambda(\bar{s}) \in U(R)$. (See McCoy [9]). Thus letting $I=R d$, we have $I \in F_{C}(R)$. Let $s^{\prime}$ denote the restriction of $\Lambda^{-1}(\operatorname{adj} \Lambda(\bar{s}))$ to $P_{R}$. Then $s^{\prime} s=\rho_{d}$ where $\rho_{d}(x)=x d$ for each $x \in P$ and since $s \in J, \rho_{d} \in J$. Let $t \in \bar{I}$. Define $t^{\prime} \in S$ by

$$
\begin{aligned}
& t^{\prime}(x)=\sum_{i, j=1}^{n} x_{j} r_{i j} f_{i}(x) \quad \text { for each } x \in P \text { where } \\
& t\left(x_{i}\right)=\sum_{j=1}^{n} x_{j} r_{i j} d \in P I \text { for } i=1, \cdots, n .
\end{aligned}
$$

Then one checks that $t=t^{\prime} \rho_{d}$ and since $\rho_{d} \in J, t \in J$. Hence $\bar{I} \leqq J$ so $J \in F(S)$ by (2.6). Therefore $F_{C}(S) \subseteq F(S)$. Thus we have shown that $F_{C}(S)=F(S)$ and by our previous remarks the theorem follows.

\section{REFERENCES}

1. H. Bass, The Morita Theorems, Lecture Notes, University of Oregon, 1962.

2. H. Cartan, and S. Eilenberg, Homological Algebra, Princeton, N. J.: Princeton University Press, 1956.

3. S. E. Dickson, A torsion theory for abelian categories, Trans. Amer. Math. Soc., 121 (1966), 223-235.

4. P. Gabriel, Des catégories abéliennes, Bull. Soc. Math. France, 90 (1962), 323-448.

5 . K. Hirata, Some types of separable extensions of rings, Nagoya Math. Journal, 33 (1968), 107-115.

6. J. P. Jans, Some aspects of torsion, Pacific J. Math., 15 (1965), 1249-1259.

7. J. Lambek, Lectures on Rings and Modules, Waltham, Mass.: Blaisdell, 1966.

8. L. Levy, Torsion-free and divisible modules over non-integral domains, Canad. J. Math., 15 (1963), 132-151.

9. N. H. McCoy, Rings and Ideals, Carus Monograph No. 8, Buffalo, N. Y., 1948.

10. K. Morita, Duality for modules and its applications to the theory of rings with minimum condition, Sci. Rep. Tokyo Kyoiku Daigaku, Sec. A, 6 (1958), 83-142.

11. F. L. Sandomierski, Semisimple maximal quotient rings, Trans. Amer. Math. Soc., 128 (1967), 112-120,

12. L. W. Small, Orders in Artinian rings, J. of Algebra, 4 (1966), 13-41.

13. E. A. Walker, and C. Walker, Quotient categories and rings of quotients. (to appear).

Received December 4, 1969. Portions of this paper are from the author's doctoral dissertation written at the University of Oregon under the direction of F. W. Anderson.

Kent State University 


\section{PACIFIC JOURNAL OF MATHEMATICS}

\section{EDITORS}

H. SAMELSON

Stanford University

Stanford, California 94305

C. R. Новву

University of Washington

Seattle, Washington 98105
J. DUGundJI

Department of Mathematics

University of Southern California

Los Angeles, California 90007

RICHARD ARENS

University of California

Los Angeles, California 90024

\section{ASSOCIATE EDITORS}
E. F. BECKENBACH
B. H. NeumanN
F. WOLF
K. YOSHIDA

\section{SUPPORTING INSTITUTIONS}
UNIVERSITY OF BRITISH COLUMBIA
CALIFORNIA INSTITUTE OF TECHNOLOGY
UNIVERSITY OF CALIFORNIA
MONTANA STATE UNIVERSITY
UNIVERSITY OF NEVADA
NEW MEXICO STATE UNIVERSITY
OREGON STATE UNIVERSITY
UNIVERSITY OF OREGON
OSAKA UNIVERSITY
UNIVERSITY OF SOUTHERN CALIFORNIA
STANFORD UNIVERSITY
UNIVERSITY OF TOKYO
UNIVERSITY OF UTAH
WASHINGTON STATE UNIVERSITY
UNIVERSITY OF WASHINGTON
AMERICAN MATHEMATICAL SOCIETY CHEVRON RESEARCH CORPORATION TRW SYSTEMS
NAVAL WEAPONS CENTER 


\section{Pacific Journal of Mathematics}

\section{Vol. 37, No. $1 \quad$ January, 1971}

Gregory Frank Bachelis and Haskell Paul Rosenthal, On unconditionally

converging series and biorthogonal systems in a Banach space .........

Richard William Beals, On spectral theory and scattering for elliptic

operators with singular potentials .........................

J. Lennart (John) Berggren, Solvable and supersolvable groups in which every element is conjugate to its inverse ........................ 21

Lindsay Nathan Childs, On covering spaces and Galois extensions ..........

William Jay Davis, David William Dean and Ivan Singer, Multipliers and

unconditional convergence of biorthogonal expansions..............

Leroy John Derr, Triangular matrices with the isoclinal property ............

Paul Erdős, Robert James McEliece and Herbert Taylor, Ramsey bounds for

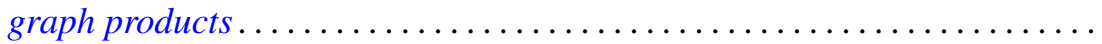

Edward Graham Evans, Jr., On epimorphisms to finitely generated

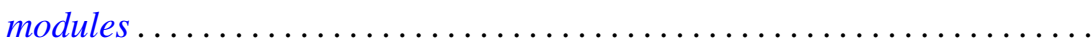

Hector O. Fattorini, The abstract Goursat problem ................. 51

Robert Dutton Fray and David Paul Roselle, Weighted lattice paths .........

Thomas L. Goulding and Augusto H. Ortiz, Structure of semiprime $(p, q)$

radicals ...........................................

E. W. Johnson and J. P. Lediaev, Structure of Noether lattices with join-principal maximal elements ....

David Samuel Kinderlehrer, The regularity of minimal surfaces defined over

slit domains

Alistair H. Lachlan, The transcendental rank of a theory. .

Frank David Lesley, Differentiability of minimal surfaces at the boundary ...

Wolfgang Liebert, Characterization of the endomorphism rings of divisible torsion modules and reduced complete torsion-free modules over complete discrete valuation rings....

Lawrence Carlton Moore, Strictly increasing Riesz norms.

Raymond Moos Redheffer, An inequality for the Hilbert transform ...

James Ted Rogers Jr., Mapping solenoids onto strongly self-entwined,

circle-like continua..........................

Sherman K. Stein, B-sets and planar maps ................... 217

Darrell R. Turnidge, Torsion theories and rings of quotients of Morita

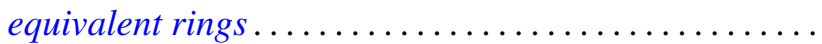

Fred Ustina, The Hausdorff means of double Fourier series and the principle of localization ................................

Stanley Joseph Wertheimer, Quasi-compactness and decompositions for arbitrary relations.

Howard Henry Wicke and John Mays Worrell Jr., On the open continuous images of paracompact $\check{C}$ ech complete spaces... 